\title{
Photonic Crystals for Confining, Guiding, and Emitting Light
}

\author{
Axel Scherer, Oskar Painter, Jelena Vuckovic, Marko Loncar, and Tomoyuki Yoshie
}

\begin{abstract}
We show that by using the photonic crystals, we can confine, guide, and emit light efficiently. By precise control over the geometry and three-dimensional design, it is possible to obtain high quality optical devices with extremely small dimensions. Here we describe examples of high- $Q$ optical nanocavities, photonic crystal waveguides, and surface plasmon enhanced light-emitting diode (LEDs).

Index Terms-Finite-difference time-domain (FDTD) methods, light-emitting diodes (LEDs), microcavities, nanooptics, photonic bandgap (PBG) materials, photonic crystal waveguides, photonic crystals, quantum-well laser, semiconductor device fabrication, spontaneous emission, surface plasmons.
\end{abstract}

\section{Photonic Crystal Nanocavities}

$\mathbf{T}$ HE PAST rapid emergence of optical microcavity devices, such as vertical-cavity surface-emitting lasers (VCSELs) [1] and [2] can be largely attributed to the high precision over the layer thickness control available during semiconductor crystal growth. High reflectivity mirrors can, thus, be grown with subnanometer accuracy to define high-Q cavities in the vertical dimension. Recently, it has also become possible to microfabricate high reflectivity mirrors by creating two- and three-dimensional periodic structures. These periodic "photonic crystals" [3] and [4] can be designed to open up frequency bands within which the propagation of electromagnetic waves is forbidden irrespective of the propagation direction in space and define photonic bandgaps. When combined with high index contrast slabs in which light can be efficiently guided, microfabricated two-dimensional photonic bandgap (PBG) mirrors provide us with the geometries needed to confine and concentrate light into extremely small volumes and to obtain very high field intensities. Here we show that it is possible to use these "artificially" microfabricated crystals in functional optical devices, such as lasers, modulators, add-drop filters, polarizers and detectors.

Fabrication of optical structures has evolved to a precision which allows us to control light within etched nanostructures [5]. For example, subwavelength nano-optic cavities can be used for efficient and flexible control over the emission wavelength [6] and [7]. Similarly, nanofabricated optical waveguides can be used for efficient coupling of light between devices. This

\footnotetext{
Manuscript received February 27, 2002; revised March 1, 2002. This work was supported in part by the Air Force Office of Scientific Research (AFOSR) under the Defense Advanced Research Projects Agency (DARPA)/Quantum Information Science and Technology (QuIST) Program, and in part by the National Science Foundation (NSF) under Grant ECS-9912039, and the Army Research Office (ARO) under a Multidisciplinary University Research Initiative (MURI) Grant DAAD 190010374.

The authors are with the California Institute of Technology (Caltech), Pasadena, CA 91125 USA.

Publisher Item Identifier S 1536-125X(02)04953-0.
}

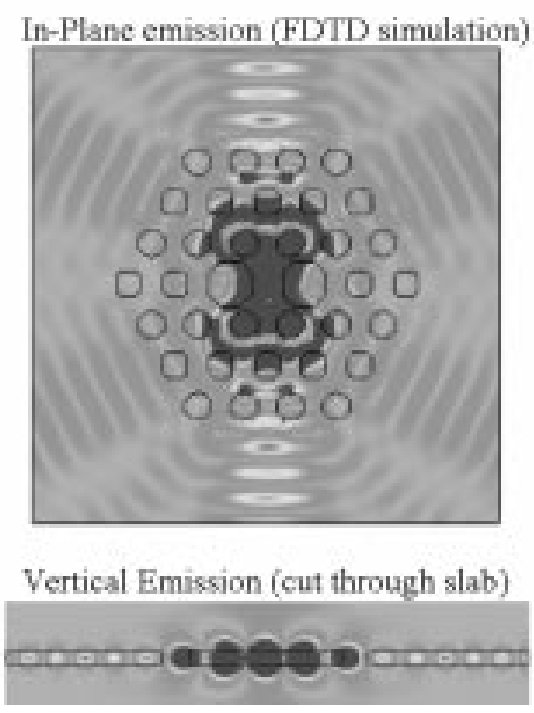

Fig. 1. Field intensity of light leakage from a single defect surrounded by three periods of photonic crystal and slightly enlarged holes in the horizontal direction. Light preferentially emits in the $y$ dipole.

new capability allows the reduction of the size of optical components and leads to their integration in large numbers, much in the same way as electronic components have been integrated for improved functionality to form microchips. As high-Q optical and electronic cavity sizes approach a cubic half-wavelength the spatial and spectral densities (both electronic and optical) increase to a point where the light-matter coupling becomes so strong that spontaneous emission is replaced by the coherent exchange of energy between the two systems [8]-[11].

We can use the lithographic control over the wavelength and polarization supported within photonic crystal cavities to construct compact nanophotonic laser (see Fig. 1) and detector arrays, as well as all-optical gates and routers. We have already demonstrated room temperature lasing in the smallest optical cavities, with mode volumes down to $2.5\left(\lambda / 2 n_{\text {slab }}\right)^{3}$, or $0.03 \mu \mathrm{m}^{3}$ in InGaAsP emitting at $1.55 \mu \mathrm{m}$. We have also been able to tune the emission wavelength of these lasers from $1450 \mathrm{~nm}$ to $1620 \mathrm{~nm}$ within a $10 \times 10$ laser array in an area of $100 \mu \mathrm{m} \times 100 \mu \mathrm{m}$ by local lithographic modification of the cavity lengths (see Fig. 2) [5]. As the mode volumes of nanocavities are decreased, the coupling efficiency between the spontaneous emission [12] within the cavity and the lasing mode can be significantly improved. We have calculated spontaneous emission coupling factors $(\beta)$ above 85\% [13] for optimized photonic crystal lasers constructed in active quantum-well (QW) material. This spontaneous emission coupling efficiency can be even higher if the linewidth of the 


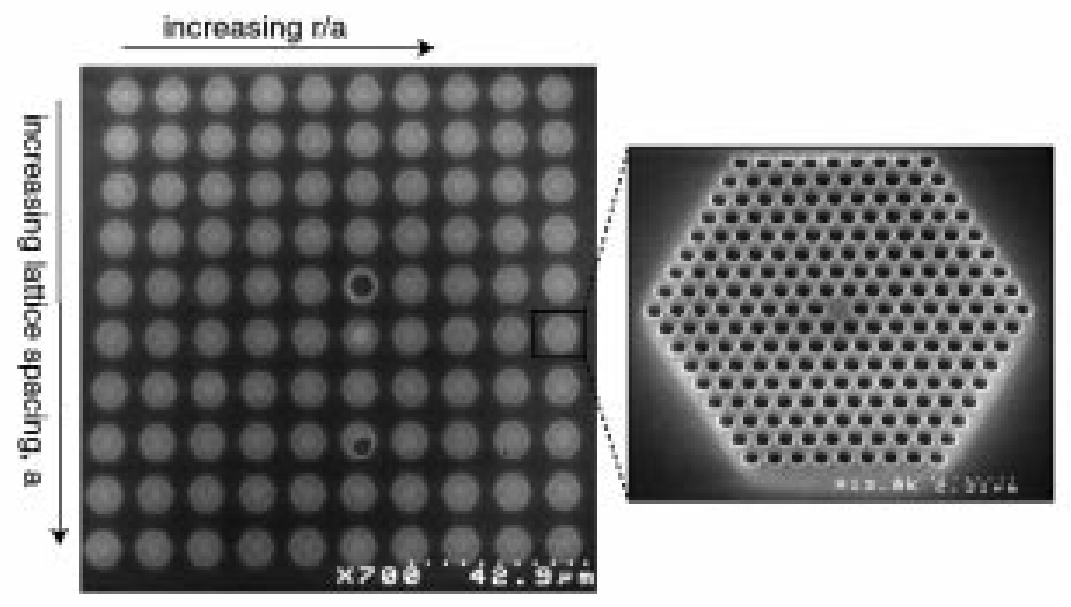

Fig. 2. $10 \times 10$ multiwavelength laser array within a $100 \times 100 \mu$ area. Each laser emits at a lithographically controlled wavelength.

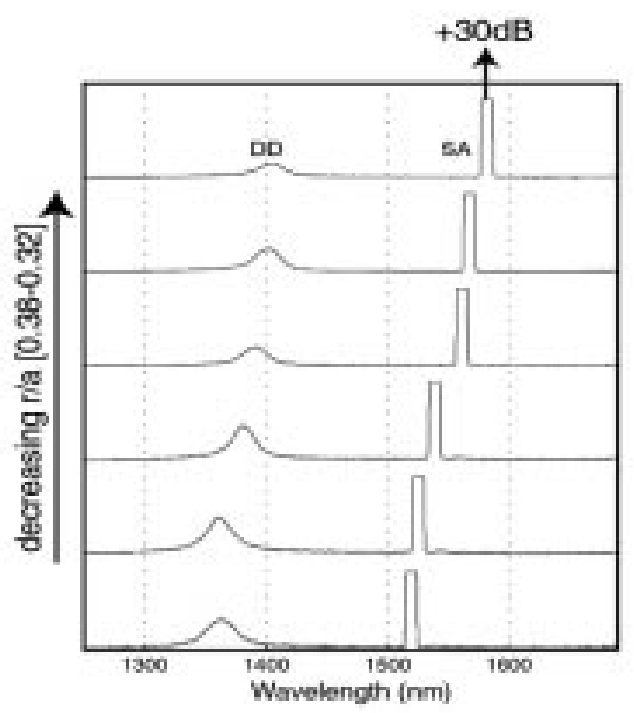

Fig. 3. Sample spectra taken from lasers defined within the $10 \times 10$ array.

semiconductor emission is narrowed, as will be the case when using quantum dot active material. Therefore, single defect photonic crystal lasers represent in many ways the ultimate evolution of VCSELs, since control over both vertical and lateral spontaneous emission is possible. With most of the spontaneous emission funneled into a single optical mode, the photonic crystal laser can be modulated at much higher frequencies even close to threshold. The photonic crystal provides us with the unique opportunity of coupling light emitted by one cavity, and using it to optically pump another with negligible diffraction losses. Photonic crystals are also the perfect medium for constructing what have been termed "photonic molecules," or interconnected cavities which can share and exchange photons. Finally, we have shown that the emission wavelength of light from these photonic crystal lasers can be varied by simple adjustments of the lithographic pattern during their fabrication. Thus, single mode lasers emitting at $1450 \mathrm{~nm}$ can be fabricated only microns apart from lasers emitting at $1600 \mathrm{~nm}$, and could

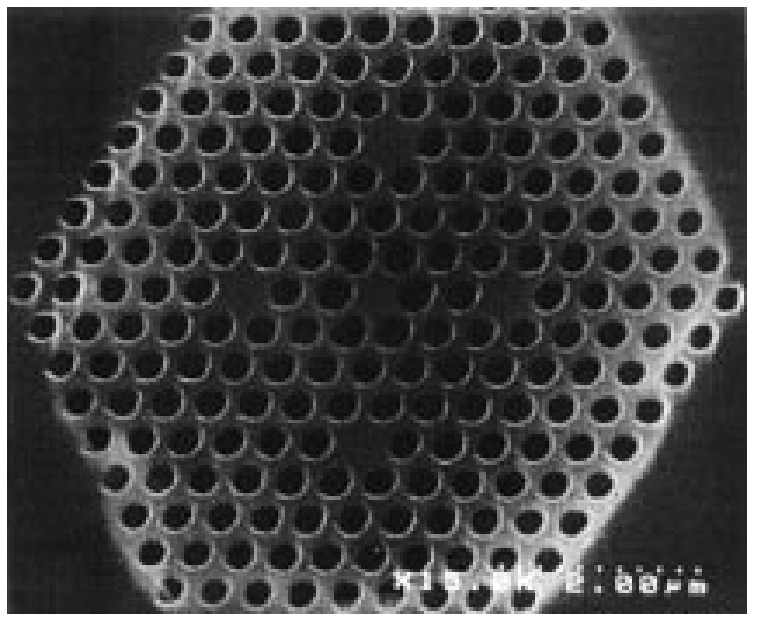

Fig. 4. Five photonic crystal cavities coupled together lithographically by arrangement in the same slab.

share the same waveguide slab. Photonic crystals provide us with the opportunity of constructing very compact laser sources with designed frequencies (Fig. 3) and polarization as well as wavelength and polarization sensitive detector arrays. Moreover, they can form very flexible platforms for connecting optical sources, detectors, routers, modulators, polarizers and filters in very compact microfabricated systems.

Another unique feature of active photonic crystal cavities (see Fig. 4), which arises from their ability to limit the number of modes supported within the laser, is the ability to build high contrast modulators.

Fig. 5 shows an example of such a single defect photonic crystal cavity, which supports a few modes within the same cavity. Depending on the diameter of the pump beam, (shown on the left-hand side of the figure), we find that different modes are excited, and these in turn exhibit different spectra. Finite-difference time-domain simulations of the expected geometric distribution of the field intensities within these modes are also shown, and the relative overlap of the pump beam with the expected mode geometries matches well to the observations. 

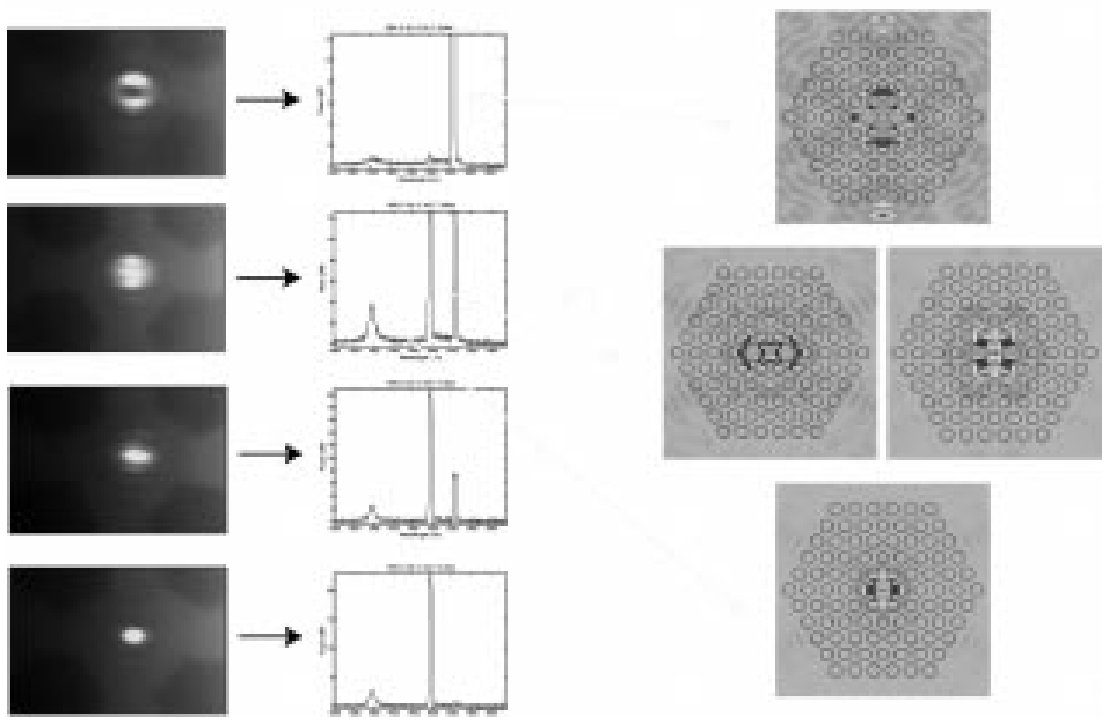

Fig. 5. Geometries of modes supported by a single defect photonic crystal cavity. We show the measured spectra and the modeled field distributions of the modes.

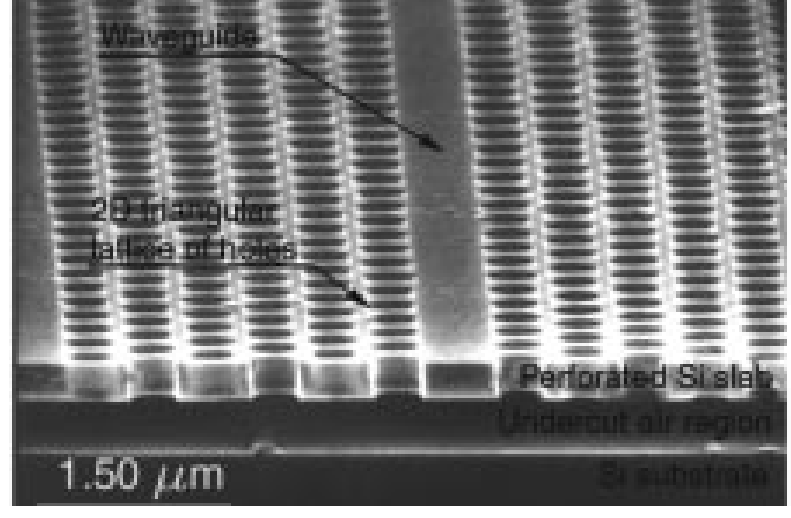

Fig. 6. Photonic crystal waveguide defined into a SOI wafer.

\section{Photonic CRystal Waveguides}

We have shown above that it is possible to generate light and modulate it with simple high-Q optical resonator cavities. However, if a nanophotonic system is to be constructed, it is also necessary to guide light from one device to another. We have conducted extensive tests on optical waveguides based on photonic crystals to explore the opportunities of using this geometry to efficiently guide light, especially around very sharp bends [18]. In the simplest geometry, a photonic crystal waveguide can be constructed by lithographically removing a line of holes from a perfect photonic crystal. This geometry provides the opportunity for light to propagate through the photonic crystal (Fig. 6) [15]-[17].

Although it is difficult to couple light into such structures, we have been able to measure light guiding in such photonic crystal waveguides through sharp bends. We have both calculated (see Fig. 8) and experimentally obtained [19] the dispersion diagram of such waveguides, and have found that several modes can propogate within them, making them less desirable for many telecommunication applications. More recently, we have developed new single mode photonic crystal waveguide designs, in which the dispersion properties can be controlled
[20]. Fig. 7 shows modified waveguide designs, fabricated in silicon-on-insulator (SOI) material, in which only one waveguiding mode is supported at desired frequency. Photonic crystal waveguides offer the opportunities to ensure mode-matching between an optical cavity and the waveguide, but much work still needs to be done to determine the dispersive character of such structures with periodic edges.

Very interesting structures can also be defined by using waveguides with periodic cavities [Fig. 7(b)], or coupled resonant cavity structures [21]. In our fabricated structures, we expect to define geometries with designed dispersive characteristics.

\section{Surface Plasmon Light Emitters}

Surface plasmons (SP), also called surface plasmons polaritons are electromagnetic surface waves that propagate along metallic surfaces, have their intensity maximum at the surface and exponentially decaying fields perpendicular to it. The condition for occurrence of such localized waves at the boundary between two media is that the two materials joined the boundary must have opposite signs of the real part of the dielectric constant (otherwise, a strong field localization at the surface cannot be obtained). This is possible at the interface between a metal operating at frequencies below the plasma frequency and any dielectric.

Numerical electromagnetic calculations in metals which are capable of manipulating radiation at the nanometer scale are very demanding. An important tool is the use of FDTD [23] and [24] methods for metal/dielectric media developed in Scherer's group to analyze theoretically the electromagnetic fields within a microcavity. In order to analyze metals as well as dielectric materials at optical frequencies, it is necessary to make certain changes [25] to Yee's standard FDTD scheme [26]. Electromagnetic fields in metals are described by adding a current term to Maxwell curl equations via a Drude model. Fig. 9 illustrates results of FDTD calculations which yield the photonic band structure and the electromagnetic field profile for a metal-clad micro- 


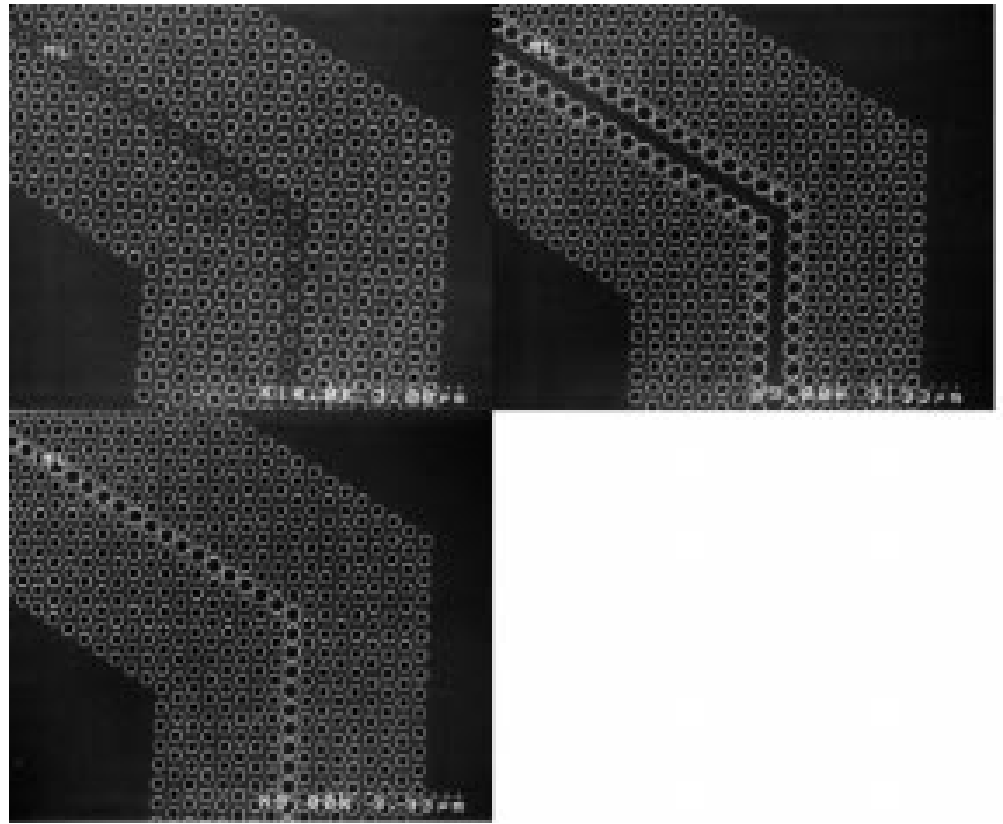

(a)

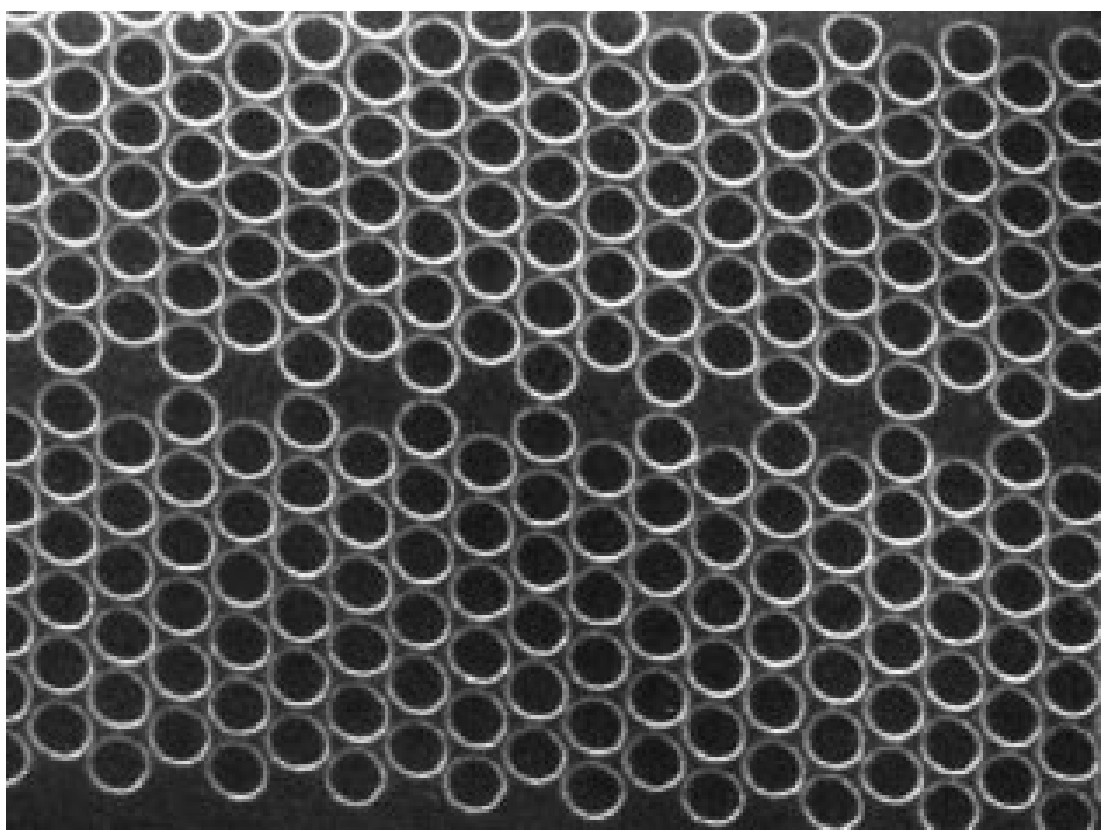

(b)

Fig. 7. (a) Novel photonic band gap (PBG) waveguide designs. (b) Coupled resonator waveguide.

cavity light-emitting diode [19]. In the frequency range where the cavity thickness is of the order of wavelength of less, coupled surface plasmon modes of the top and bottom surfaces can exist and can be used to efficiently extract light from the semiconductor slab. Very small discretization steps of $\sim 1 \mathrm{~nm}$ must be used for the finite difference algorithm to describe the propagation of electromagnetic waves in metals, and this in turn requires large computational volumes. We have developed distributed FDTD algorithms which are supported on multiprocessor computer clusters to manage the large memory requirements.

\section{SP ENHANCED LIGHT-EMITTING DIODES (SP-LEDS)}

Much scientific work has been focused on improving the extraction efficiency of light emitting diodes (LEDs). Many interesting approaches have been proposed to accomplish this, such as the use of thin light emitting layers with surface texturing, resonant cavities or photon recycling. External quantum efficiencies of $31 \%$ were achieved by employing reflection from a bottom metal mirror together with a textured top semiconductor surface. Apart from efforts to improve light extraction from a 


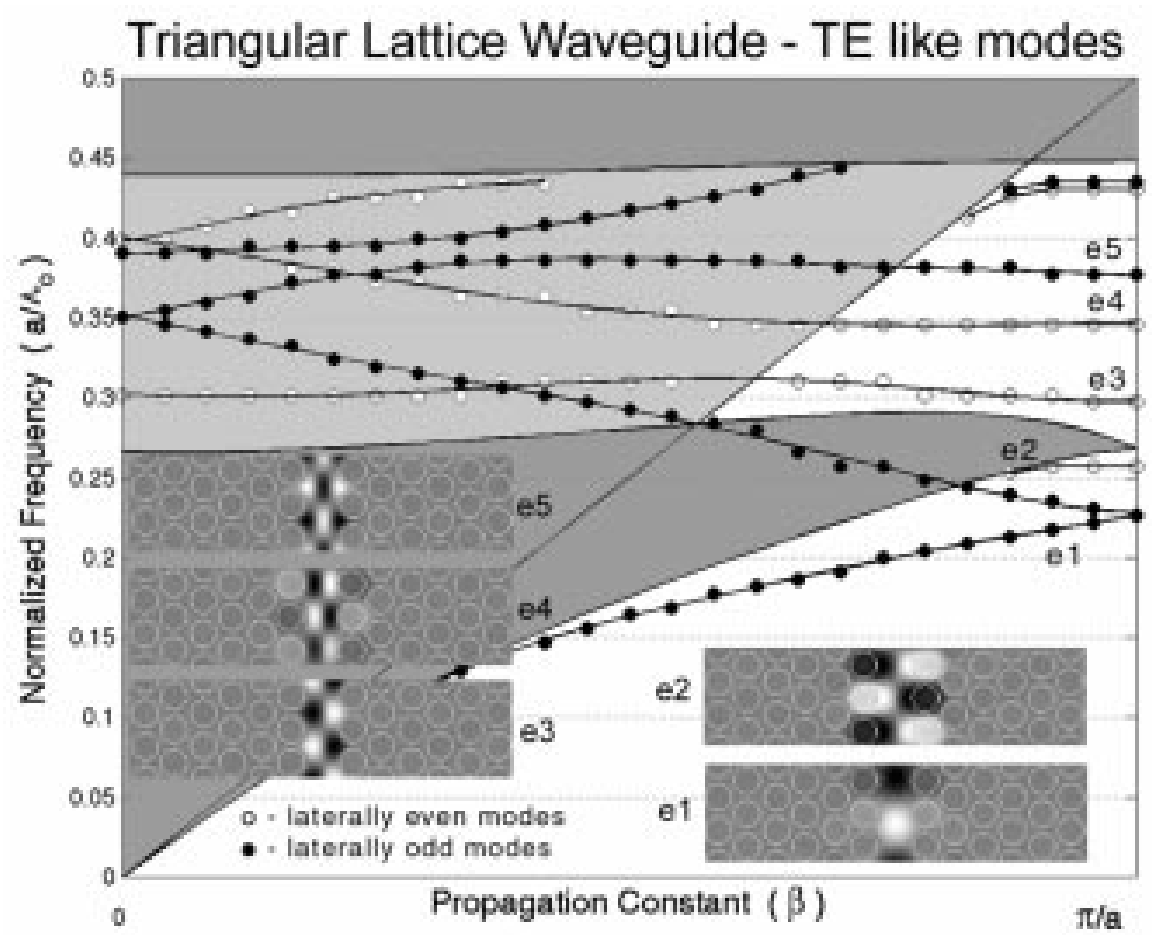

Fig. 8. Dispersion diagram for vertically even TE-like guided modes in the single-line defect PBG waveguide based on the triangular lattice. Insets show field patters of $\mathbf{B}_{\mathbf{z}}$ of a surface plasmon wave.

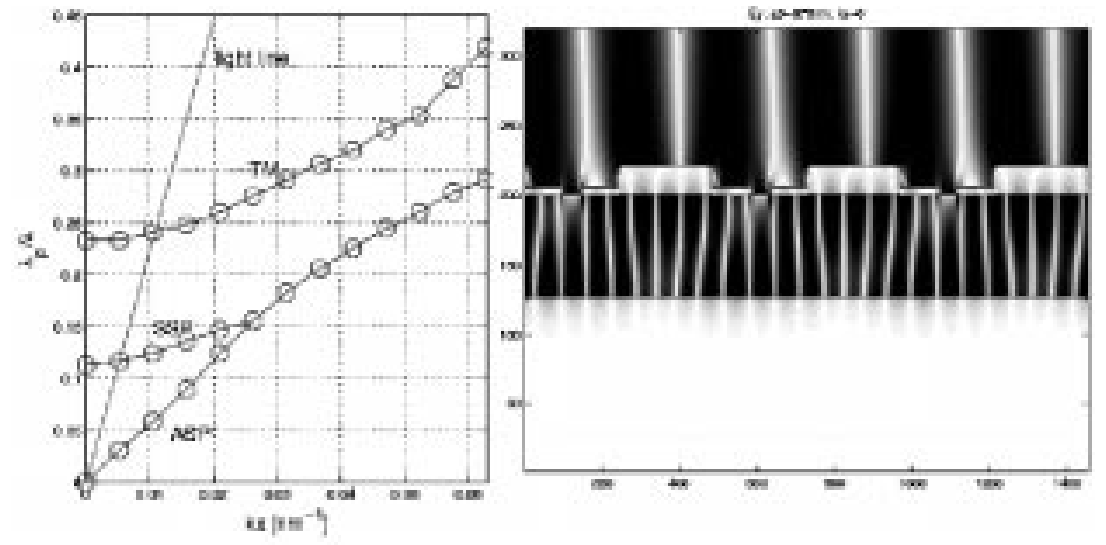

Fig. 9. Band diagram (left) and transverse magnetic (TM) field profile (right) for p-polarized light of a metal clad microcavity. The core is assumed to be made of 150-nm thick GaAs (refractive index $n=3.5$ ). Top and bottom metal layers are made of silver with the following parameters: $h \omega_{p}=8.8 \mathrm{eV}$ and $h v=0.05 \mathrm{eV}$. The top layer is only 40-nm thick and the bottom layer is $200 \mathrm{~nm}$ thick. The analyzed structure is surrounded by air on top and bottom and the slab is infinite in lateral dimension. Only the bottom three bands are shown, since they are within the frequency range of interest.

semiconductor device, it is also possible to enhance the light emission rate within a semiconductor. This approach is based on Purcell's prediction in 1946 [12] that the radiation rate of an atom placed within a wavelength-sized cavity can be changed. A 12-fold enhancement of spontaneous emission was recently measured in a semiconductor optical microcavity at low temperatures. Metallic structures were identified as candidates for very large decay rate enhancement-much larger than the one achievable by semiconductor photonic crystals. Unfortunately, much of the previous work on surface plasmon emitters was limited by the need to efficiently extract the light generated within such structures.

In order to build an ideal, highly efficient LED, it is desirable to improve both the extraction efficiency and simultane-

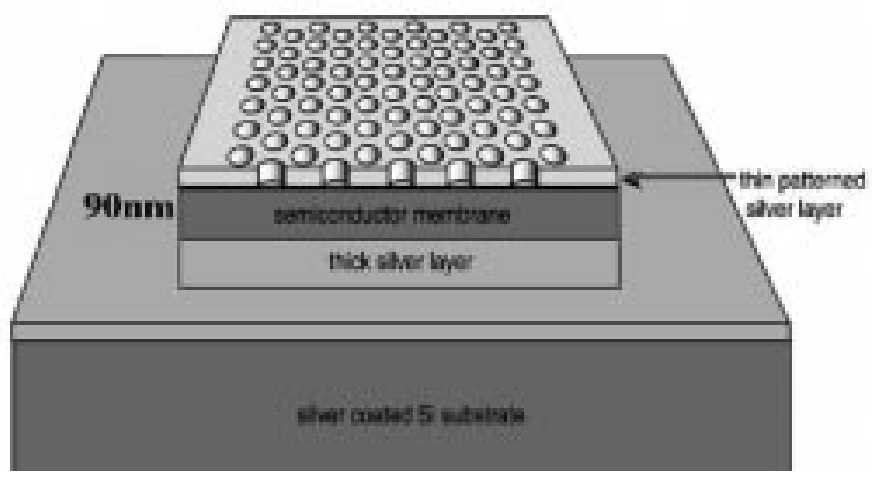

Fig. 10. Design of the surface plasmon LED structure. 


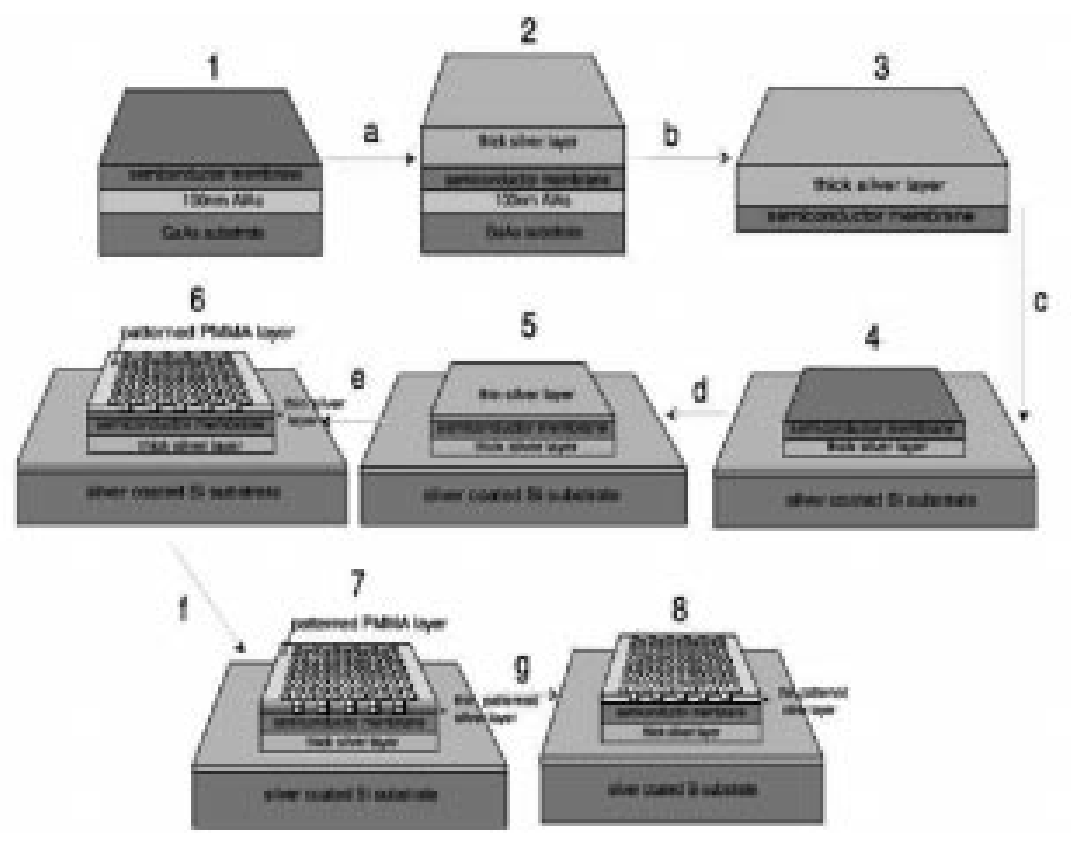

Fig. 11. Fabrication sequence for constructing a SP-LED.

ously enhance the spontaneous emission rate. Metallic photonic crystals have attracted significant scientific attention in recent years for both of these purposes. In 1996, Bill Barnes at the University of Exeter observed a PBG for surface modes on a silver surface textured with a hexagonal array of dots. Two years later, researchers at NEC and their collaborators observed sharp peaks in the transmission of light through a metallic layer patterned with a hexagonal array of subwavelength holes. Both experimental results were attributed to the peculiar properties of surface plasmons polaritons-modes that exist at the interface of two media with opposite signs of the real part of dielectric constant.

In order to couple the emission to SP waves from a single metal-semiconductor surface, it is typically necessary to position the QW very close to that metal surface and within the fringing field depth of the SP. However, the nonradiative transfer between a QW and the metal increases as their distance decreases. The question is then how much of the observed large reduction in lifetime at small distances is due to the coupling to SP waves, and what portion of it comes from other nonradiative processes. The only part that can be possibly extracted outside of the structure by properly designing an "antenna" (i.e., by structuring the surface of metal) is the portion coupled to SP waves. To reduce the nonradiative transfer, we decided to position the QW further from the metal surface (at distances of 40 to $50 \mathrm{~nm}$ ). However, the intensity of SP waves decreases exponentially as a function of distance from the metallic surface. In order to increase the field intensity at the position of the QW even more, it is possible to use the coupled surface plasmon modes of two closely spaced metallic interfaces.

The analyzed structure consists of a 90-nm thick-semiconductor layer sandwiched between two silver films. A single $8 \mathrm{~nm}$ thick InGaAs quantum well is positioned in the middle of the semiconductor membrane. The fabrication sequence is summarized in Fig. 11 and a scanning electron micrograph of

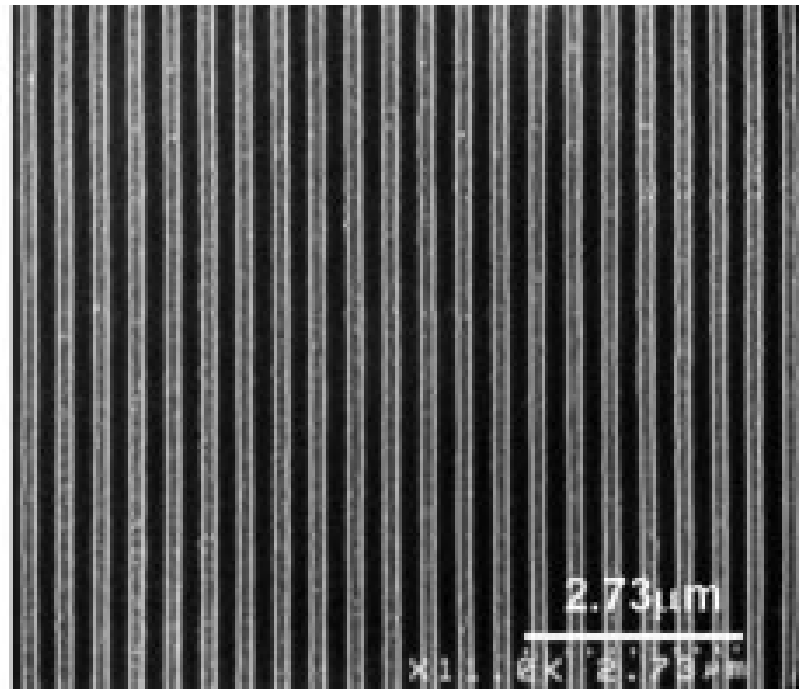

Fig. 12. Scanning electron micrograph of a surface plasmon enhanced LED showing the metal grating pattern which is used to couple out the radiation.

a completed surface plasmon LED is shown in Fig. 12. The main emission peak is at the wavelength of $990 \mathrm{~nm}$, corresponding to conduction-to-heavy hole $(\mathrm{C}-\mathrm{HH})$ band transitions. Another peak can be observed at $930 \mathrm{~nm}$, corresponding to conduction-to-light hole ( $\mathrm{C}-\mathrm{LH})$ band transitions. If a periodic pattern is defined in the top semitransparent metal layer by lithography (Fig. 10), it is possible to efficiently couple out the light emitted from the semiconductor and to simultaneously enhance the spontaneous emission rate. For the analyzed designs, we theoretically estimate extraction efficiencies as high as $37 \%$ and Purcell factors $(F p)$ of up to 4.5 [22]. We have experimentally measured photoluminescence intensities of up to 46 times higher in fabricated structures compared to unprocessed wafers. The increased light emission is due to an increase in the efficiency and an increase in the pumping intensity resulting 


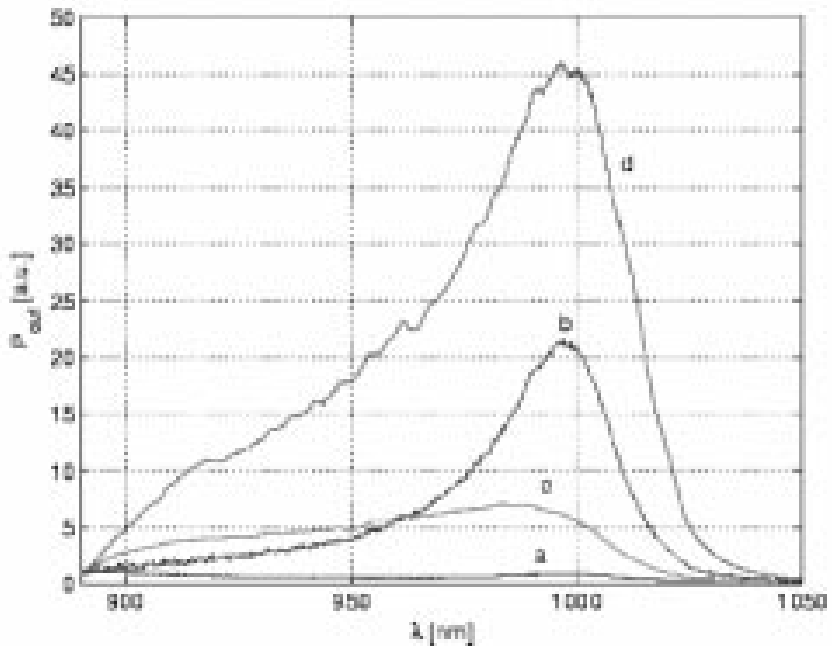

Fig. 13. Luminescence spectra from a surface plasmon enhanced LED at various stages of fabrication (see text).

TABLE I

PARAMETERS OF GRATINGS USED FOR EXTRACTING THE EMISSION FROM THE Metal Clad Microcavity. $a$ Is the Grating Periodicity, $s$ Is the GaP BETWEen Silver StRIPES, $\theta$ Is the ANGLE OF THE OUtCOUPLED PHOTON WITH RESPECT TO NORMAL, AND $\eta$ IS THE NORMALIZED DIFFRACTION EFFICIENCY INTO THE $n$ TH ORDER OF A GRATING. THE OUTCOUPLING HAPPENS THROUGH THE $n$ TH ORDER OF A GRATING

a [nm] $n \theta($ at $986 \mathrm{~nm}) \quad \theta($ at $930 \mathrm{~nm})$ s[nm] $\eta$

\begin{tabular}{llllll}
\hline 250 & -1 & $6^{\circ}$ & $25^{\circ}$ & 160 & 0.82 \\
480 & -2 & $3.5^{\circ}$ & $15.3^{\circ}$ & 160 & 0.19 \\
650 & -3 & $30^{\circ}$ & $9^{\circ}$ & 160 & 0.06 \\
650 & -3 & $30^{\circ}$ & $9^{\circ}$ & 100 & 0.11
\end{tabular}

from trapping of pump photons within the microcavity. The measured photoluminescence spectra are shown in Fig. 13. The spectra labels correspond: (a) the unprocessed wafer; (b) the half-processed wafer (i.e., 90-nm-thick semiconductor membrane on top of a thick, nontransparent silver layer); (c) the unpatterned metal-clad microcavity (i.e., a semiconductor membrane sandwiched between two metal films, without patterning of the top silver layer); and (d) the fully processed structure (where silver stripes are defined in the top silver layer, with the grating periodicity of $250 \mathrm{~nm}$ and the $160 \mathrm{~nm}$ gap between silver stripes). From the calculated values shown in Table I, we conclude that the grating with periodicity of $250 \mathrm{~nm}$ will have the best performance, since it has the largest diffraction efficiency. The grating with periodicity of $650 \mathrm{~nm}$ and a gap between stripes of $s=160 \mathrm{~nm}$ will have the worst performance, which can be somewhat improved by reducing the gap to $100 \mathrm{~nm}$.

For all fully processed wafers, including the unpatterned metal clad microcavity, the full-width at half-maximum (FWHM) of the emission spectra is in the range of 60 to $110 \mathrm{~nm}$. Therefore, their quality factors are between 10 and 15 . For the half-processed wafer, a FWHM is $32 \mathrm{~nm}$. Because of a bulk emission tail at lower wavelengths, a luminescence peak at $930 \mathrm{~nm}$ for unprocessed wafers cannot be clearly resolved. The spectra of unprocessed, half-processed and fully processed

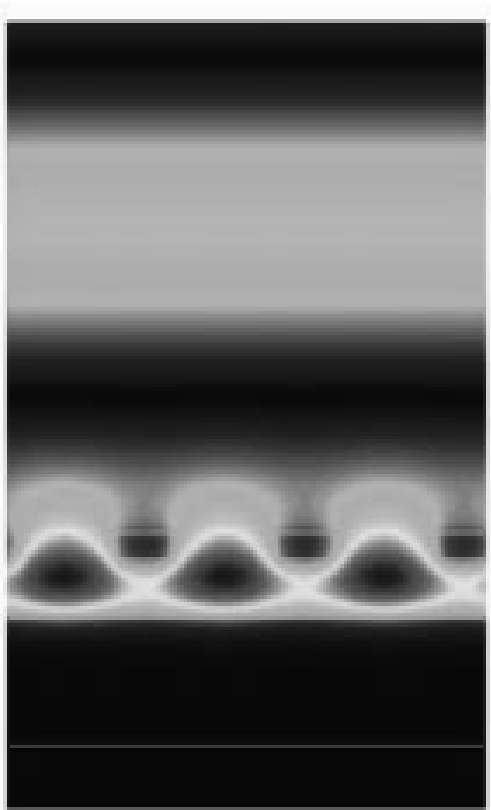

Fig. 14. FDTD calculation of TE field intensities emitted from a surface plasmon LED with a 250-nm-period grating. Collimated light emission is observed in the time evolution of the field.

wafers are shown in Fig. 13. The PL peak of the unprocessed wafer at $986 \mathrm{~nm}$ was normalized to 1 . A GaAs filter was applied in front of the detector to cutoff wavelengths below $890 \mathrm{~nm}$. As can be seen from the luminescence curves, it is possible to increase the luminescence intensity of a diode 48 times over a grown heterostructure by introducing a metal clad waveguide with a semitransparent patterned surface plasmon extractor. More interestingly, when comparing curve (b), the half-processed wafer on top of a silver mirror, with curve (d), the fully processed and metalized structure, much higher light extraction is obtained through the top metal layer. Such a structure is ideally suited for electrical pumping, since the metal layers can be used for both light extraction as well as carrier injection. Moreover, our calculations predict that efficient collimated light emission can be generated from surface plasmon LEDs (Fig. 14). Here we propose to optimize this diode structure for electrical pumping, which is expected to lead to highly efficient LEDs with very fast modulation speeds. Our preliminary tests have indicated that in our structures, the spontaneous emission rate is increased by approximately five fold (at room temperature) over conventional LED material.

\section{CONCLUSION}

Microfabricated structures with dimensions smaller than the wavelength of light allow the efficient confining and guiding of light. High index contrast systems allow such structures to be defined with very small sizes, and, thus, leads to integration of several optical components into more functional systems. Although more complex three-dimensional geometries will eventually be developed [27], one very promising geometry is the two-dimensional photonic crystal, into which cavities, waveguides and dispersive elements can be embedded. The most important advantage of this geometry lies in the opportunity to 
lithographically couple devices together with minimal diffraction losses and excellent coupling efficiencies. We believe that photonic crystals will be extremely useful for the definition of integrated optical systems, as well as for the definition of extremely small optical cavities, which would be applicable toward strong coupling experiments with quantum dot or atom emitters. On the other hand, metallic structures with high index contrast offer the opportunity to harness surface plasmons, and we show that even with a very simple design it is possible to construct very efficient surface LEDs.

\section{REFERENCES}

[1] J. L. Jewell, J. P. Harbison, A. Scherer, Y. H. Lee, and L. T. Florez, "Vertical cavity surface emitting lasers: Growth, design, fabrication, measurements," IEEE J. Quantum Electron., vol. 27, p. 1332, 1991.

[2] A. Scherer, J. L. Jewell, and J. P. Harbison, "Lasing in sub-micron wide vertical cavity microlasers," Opt. Photonics News, vol. 2, p. 9, 1991.

[3] E. Yablonovitch, "Inhibited spontaneous emission in solid state physics and electronics?," Phys. Rev. Lett., E, vol. 58, no. 20, p. 2059, 1987.

[4] S. John, "Strong localization of photons in certain disordered dielectric superlattices," Phys. Rev. Lett., vol. 58, no. 23, p. 2486, 1987.

[5] O. Painter et al., "Lithographic tuning of a two-dimensional photonic crystal laser array," IEEE Photonic Tech. L, vol. 12, no. 9, pp. 1126-1128, Sept. 2000.

[6] O. Painter et al., "Room temperature photonic crystal defect lasers at near-infrared wavelengths in InGaAsP," J. Lightwave Technol., vol. 17, no. 11, pp. 2082-2088, Nov. 1999.

[7] T. Yoshie, S. Scherer, H. Chen, D. Huffaker, and D. Deppe, "Optical chasracterization of two-dimensional photonic crystal cavities with indium arsenide quantum dot emitters," Appl. Phys. Lett., vol. 79, no. 1, pp. 114-116, July 2001

[8] H. Yokoyama, "Physics and device applications of optical microcavities," Science, vol. 256, pp. 66-70, 1992.

[9] Y. Yamamoto and S. Machida, "Microcavity semiconductor laser with enhanced spontaneous emission," Phys. Rev. A, Gen. Phys., vol. 44, no. 1, p. 657,1991

[10] L. A. Graham, D. L. Huffaker, and D. G. Deppe, "Spontaneous lifetime control in a native oxide apertured microcavity," Appl. Phys. Lett., vol. 74, no. 17 , p. 2408, 1999.

[11] G. P. Agrawal and N. K. Dutta, Semiconductor Lasers. New York: Van Nostrand, 1993

[12] E. M. Purcell, "Spontaneous emission probabilities at radio frequencies," Phys. Rev., vol. 69, p. 681, 1946.

[13] J. Vuckovic, O. Painter, Y. Xu, A. Yariv, and A. Scherer, "Finite difference time domain calculations of the spontaneous emission coupling factor in optical microcavities," IEEE J. Quantum Electron., vol. 35, pp. 1168-1175, Aug. 1999.

[14] O. Painter, R. K. Lee, and A. Scherer, "Two-dimensional photonic band-gap defect mode laser," Science, vol. 284, no. 5421, pp. 1819-1821, June 11, 1999.

[15] M. Loncar et al., "Design and fabrication of silicon photonic crystal optical waveguides," J. Lightwave Technol., vol. 18, pp. 1402-1411, Oct. 2000.

[16] J. D. Joannopoulos, R. D. Meade, and J. N. Winn, Photonic Crystals. Princeton, NJ: Princeton Univ. Press, 1995.
[17] S.-Y. Lin, E. Chow, V. Hietala, P. R. Villeneuve, and J. D. Joannopoulos, "Experimental demonstration of guiding and bending of electromagnetic waves in a photonic crystal," Science, vol. 282, p. 274, 1998.

[18] M. Loncar, D. Nedeljkovic, and T. Doll, "Waveguiding in planar photonic crystals," Appl. Phys. Lett., vol. 77, no. 13, pp. 1937-1939, 1999.

[19] M. Loncar et al., "Experimental and theoretical confirmation of Block-mode light propagation in planar photonic crystal waveguides," Appl. Phys. Lett., vol. 80, no. 10, pp. 1689-1691, Mar. 2002.

[20] M. Loncar, J. Vuckovic, and A. Scherer, "Methods for controlling positions of guided modes of photonic-crystal waveguides," J. Opt. Soc. Amer. B, Opt. Phys., vol. 18, no. 9, pp. 1362-1368, Sept. 2001.

[21] A. Yariv et al., "Coupled-resonator optical waveguide: A proposal and analysis," Opt. Lett., vol. 24, no. 11, pp. 711-713, June 1, 1999.

[22] J. Vuckovic, M. Loncar, and A. Scherer, "Surface plasmon enhanced light-emitting diode," IEEE J. Quantum Electron., vol. 36, pp. 1131-1144, Oct. 2000

[23] B. D'Urso, O. Painter, J. O'Brien, T. Tombrello, A. Yariv, and A. Scherer, "Modal reflectivity in finite-depth 2-D photonic crystal microcavities," J. Opt. Soc. Am. B, Opt. Phys., vol. 15, no. 3, p. 1155 , 1998.

[24] A. Scherer et al., "InGaAsP photonic band gap crystal membrane microresonators," J. Vac. Sci. Technol. B, Microelectron., vol. 16, no. 6 , pp. 3906-3910, Nov.-Dec. 1998.

[25] S. A. Cummer, "An analysis of new and existing FDTD methods for isotropic cold plasma and a method for improving their accuracy," IEEE Trans. Antennas Propagat., vol. AP45, p. 392, 1997.

[26] K. S. Yee, "Numerical solution to initial boundary value problems involving Maxwell's equations in isotropic media," IEEE Trans. Antennas Propagat., vol. AP14, p. 302, 1966.

[27] C. C. Cheng et al., "Nanofabricated three dimensional photonic crystals operating at optical wavelengths," Phys. Scr., vol. T68, pp. 17-20, 1996.

Axel Scherer, photograph and biography not available at the time of publication.

Oskar Painter, photograph and biography not available at the time of publication.

Jelena Vuckovic, photograph and biography not available at the time of publication.

Marko Loncar, photograph and biography not available at the time of publication.

Tomoyuki Yoshie, photograph and biography not available at the time of publication. 